\title{
Using potential master regulator sites and paralogous expansion to construct tissue-specific transcriptional networks
}

\author{
Martin Haubrock, Jie Li, Edgar Wingender \\ From 23rd International Conference on Genome Informatics (GIW 2012) \\ Tainan, Taiwan. 12-14 December 2012
}

\begin{abstract}
Background: Transcriptional networks of higher eukaryotes are difficult to obtain. Available experimental data from conventional approaches are sporadic, while those generated with modern high-throughput technologies are biased. Computational predictions are generally perceived as being flooded with high rates of false positives. New concepts about the structure of regulatory regions and the function of master regulator sites may provide a way out of this dilemma.
\end{abstract}

Methods: We combined promoter scanning with positional weight matrices with a 4-genome conservativity analysis to predict high-affinity, highly conserved transcription factor (TF) binding sites and to infer TF-target gene relations. They were expanded to paralogous TFs and filtered for tissue-specific expression patterns to obtain a reference transcriptional network (RTN) as well as tissue-specific transcriptional networks (TTNs).

Results: When validated with experimental data sets, the predictions done showed the expected trends of true positive and true negative predictions, resulting in satisfying sensitivity and specificity characteristics. This also proved that confining the network reconstruction to the 1\% top-ranking TF-target predictions gives rise to networks with expected degree distributions. Their expansion to paralogous TFs enriches them by tissue-specific regulators, providing a reasonable basis to reconstruct tissue-specific transcriptional networks.

Conclusions: The concept of master regulator or seed sites provides a reasonable starting point to select predicted TF-target relations, which, together with a paralogous expansion, allow for reconstruction of tissuespecific transcriptional networks.

\section{Background}

Regulation of transcription is mediated through complex arrays of transcription factor binding sites (TFBSs), which constitute promoter and enhancer regions. In spite of the advent of high-throughput approaches to identify TFBSs in a given cellular context, the available information, most comprehensively collected in the TRANSFAC $^{\circledR}$ database [1], is still fragmented and biased with regard to the systems selected. Consequently, any transcriptional network reconstructed from the available experimental data is highly incomplete. This situation

\footnotetext{
* Correspondence: Edgar.Wingender@bioinf.med.uni-goettingen.de Department of Bioinformatics, University Medical Center Göttingen, Goldschmidtstrasse 1, D-37077 Göttingen, Germany
}

deteriorates further when filtering such a transcriptional "reference" network for gene expression data in order to generate tissue-specific networks. Therefore, constructing comprehensive gene regulatory networks still depends on reliable algorithms for predicting individual TFBSs as a basis for inferring TF-target gene relations. These predictions, however, depend on the availability of information about the DNA-binding specificity of ideally all TFs encoded by a genome. Unfortunately, we are far from this ideal situation, so that we can do such predictions only for a subset of, e.g., human TFs. Although promising methods have been reported for inferring DNA-binding specificities by homology modeling [2,3], the required 3D 
structures of TF-DNA complexes are known for only a minority of factors.

Recent studies have applied high-throughput approaches to map active promoters and enhancers in a particular cellular context by capturing epigenetic characteristics such as specific histone methylation patterns [4]. However, it still has to be revealed what the exact regulation of a given gene is, i.e. which functional TFBSs are there in its regulatory regions, and which is the original signal that flags a promoter region as such. Conceivably, the recently published concepts about master transcription factors [5] or pioneer transcription factors [6] may provide a clue to this problem.

In this study, we started from the following related working model as hypothesis: In the genome of a given higher eukaryotic cell, promoter sequences have to be "flagged" in order to be recognizable by the transcription machinery. Each of these flags is realized by a highaffinity TFBS, which, due to its functional importance, is generally conserved among genomes that are phylogenetically not too distant. These high-affinity and conserved sites serve as nucleation centers, or "seeds", to govern the proper assembly of TFs at one promoter, which also involves a set of additional transcription factors with binding sites of decreasing affinity and acting in a concomitantly optional manner.

\section{Methods \\ TFBS prediction}

We started from 35,750 RefSeq-annotated human promoter regions (UCSC track refGene, Apr. 14, 2010, hg19) which are linked to 21,532 unique genes. We selected the $1-\mathrm{kb}$ upstream regions based on the RefSeq annotation to cover the corresponding human promoter regions. We retrieved ortholog promoter regions from mouse, dog, and cow genomes from the 46_WAY_MULTIZ_hg19 whole genome alignments provided by UCSC for 46 vertebrates using UCSC/Galaxy [7]. The corresponding sequence builds are hg19, mm9, canFan2, and bosTau4. Gaps resulting from the multiple genome alignment were removed from the promoter sequences. Potential transcription factor binding sites (TFBS) were then identified using all available vertebrate matrices (854 PWM) of the TRANSFAC matrix library (release 2009.4) and the program $\mathrm{Match}^{\mathrm{TM}}$ [8]. We applied all vertebrate matrices using default minFN ("minimize false negatives") thresholds in order to retrieve almost all potential transcription factor binding sites that have at least the quality of the used TFBS which are given in the corresponding matrix [8]. The predictions were then mapped back to the whole genome alignments. We next filtered for conserved TFBS predictions: a conserved TFBS has to start or end at a non-gap symbol in the corresponding promoter alignment. Finally we ranked all conserved TFBSs according to their
Match score and selected the top-ranking 1\%, 2\%, 3\%, 5\%, etc for evaluations. The $100 \%$ profile comprises all conserved TFBSs that were identified with minFN thresholds. For further analyses of the network characteristics, the top-ranking $1 \%$ predicted binding sites for each matrix were used.

\section{From predictions to gene regulatory network}

Using the TRANSFAC library we ended up with a list of predicted transcription factor binding sites related to the TRANSFAC matrix identifiers. To build gene regulatory networks we translated these matrix identifiers, which are linked to lists of related species-specific proteins, to official human gene symbols.

For "paralogous expansion", we used our new Human Transcription Factor Classification to construct gene regulatory networks (http://www.bioinf.med.uni-goettingen.de/ projects/tfclassification/). This collection classifies human transcription factors into families and subfamilies mainly based on the sequence similarities of their DNA-binding domains (DBDs). Since at the lowest classification level, the DBDs are usually extremely similar, the DNA-binding specificities can be assumed to be nearly identical as well. We therefore expanded all TF-target links to all members of the corresponding TF (sub-)family, for which no matrix is as yet available.

\section{Evaluation of conserved binding site prediction}

The verification of the predicted binding sites was done using experimentally identified regulatory regions from the Encode project [9]. ENCODE provides a regulatory super-track as a downloadable file. This archive is summarizing all transcription factor ChIPseq experiments which have been done within the ENCODE project based on the human genome build 37 (hg19). Altogether whole genome binding sites and their genomic coordinates are available for more than 140 different human transcription factors. They were used to evaluate our TFBS and the inferred TF-target predictions by computing the True Positive (TP), False Positive (FP), False Negative (FN), and True Negative (TN) rates for some human transcription factors. If a predicted TFBS is found in a ChIP-seq region as well, we count it as a TP. If a TFBS prediction is not detected by a ChIPseq experiment this is an FP result. An FN result is obtained when a ChIPseq region is overlapping with a potential promoter region (including the fragment of overlapping the promoter regions at least with 500 nucleotides), but we don't predict a TFBS for this situation. A TN result is related to a situation, where we neither predicted a TFBS nor a ChIPseq region was found. Using these statistical measurements we determine the Positive Predictive Value (or precision; $\mathrm{PPV}=\mathrm{TP} /$ $(\mathrm{TP}+\mathrm{FP}))$, Specificity $(\mathrm{Spec}=\mathrm{TN} /(\mathrm{TN}+\mathrm{FP}))$, and the True- 
Positive-Rate $(\mathrm{TPR}=\mathrm{TP} /(\mathrm{TP}+\mathrm{FN})$; also: sensitivity or recall) to detect the accuracy of a ChIP-seq evaluation.

\section{Tissue-specificity of transcription factors}

Based on UniGene [10] we have downloaded the gene expression profiles for 8 different tissues: brain, heart, kidney, liver, ovary, prostate, spleen, testis.

\section{Results \\ Reconstruction of the transcriptional network through predicted TFBSs}

Previous studies have shown that sequence conservation can improve transcription factor binding site predictions $[11,12]$. Therefore, we combined standard PWM scanning with a four species conservation filtering to identify potential TFBSs and, on this basis, to infer TF-target gene relations for a comprehensive reference transcriptional network (RTN). With this strategy (see Methods for details), we predicted $4,3 * 10 \mathrm{e} 7$ TFBS which are conserved among these four species (hg19, mm9, canFam2, bosTau4). These predictions are linked to 16,900 unique human gene symbols. $47.3 \%$ of all human promoters (35,750 RefSeqannotated human promoter regions) share at least one conserved predicted binding site with the mouse, dog, and cow species. When selecting only the best $1 \%$ predictions of each TRANSFAC matrix we found that 15,619 genes (43.7\%) share a conserved, high-scoring binding site. Altogether, we ended up with 490,277 TFBS predictions.

\section{Paralogous expansion of the transcription network}

We used a fundamentally revised version of an earlier transcription factor classification, based on their DNAbinding domains [13], to identify groups of TFs that share DNA-binding specificity to the largest extent possible. They may be regarded as paralogs, resulting from early gene duplication events (Wingender, manuscript in preparation). This classification scheme comprises four abstraction levels: superclass, class, family, and (optionally) subfamily. Whenever one member of a bottommost clade (family or subfamily) has a TRANSFAC matrix associated, all potential binding sites and, thus, target genes predicted for this TF were copied to all other clade members. This expansion of the transcriptional reference network led to an increase of the TF genes from 442 to 742 (by $67.9 \%$ ), and increased the number of directed edges in the network from 277,661 to 728,667 (by 162.4\%) (Additional file 1, first line of the table). The expansion approach was also cross-validated for those cases where distinct (sub)family members had different TRANSFAC matrices associated (data not shown).

\section{Validation of the reconstructed network}

For 22 different transcription factors we investigated the performance of our predictions for 20 different quality levels by gradually increasing the percentile of highest scoring potential TFBSs accepted, ranking from the $1 \%$ best sites down to $100 \%$, which are all conserved sites that could be predicted with the minFN threshold. For a number of TFs, for which experimental ChIPseq data were available at ENCODE, we determined the Positive Predicted Value (PPV), the True Positive Rate (TPR) and the Specificity for the whole range of quality levels (from the $1 \%$ best predictions to $100 \%$ of all conserved TFBSs; see Methods section for details). Figure 1 is demonstrating these three values for transcription factor NF- $\kappa \mathrm{B}$ (NFKB; see Additional File 2 for all plots). For all 22 TFs studied so far, we observed a very high specificity for the 1\% selection. For E2F1, E2F4, and E2F6, the specificity was about $80 \%$, whereas it was clearly above $90 \%$ for all other 19 TFs (BRCA1, CTCF, ELF1, ETS1, FOXA1, GATA1, GATA2, GATA3, HEY1, IRF1, IRF3, NANOG, NFKB, PAX5, POU5F1, RXRA, SP1, TFAP2A, YY1). For less stringent predictions, i.e. when proceeding towards the $100 \%$ level, the specificity decreases continuously. In contrast, the TPR (sensitivity) is increasing from $1 \%$ to $100 \%$ selection. We observed heterogeneous profiles for the PPV, with usually the highest value for the $1 \%$ profile (up to $\sim 82 \%$ in the case of CTCF or $\sim 65 \%$ for YY1). In some cases, reducing the stringency of filtering led to a disproportionate increase of the TP and, thus, to an increase of the PPV. This indicates that a very high number of "real" sites are "suboptimal", i.e. match with the matrix/matrices used only at relatively low scores. In the respective contexts, these sites may have evolved to exhibit only moderate affinity instead of strongest conceivable binding. Some TFBS predictions show relatively low PPVs, which may be regarded as high numbers of false positives. However, this perception will be challenged by further investigations (see next paragraph).

Altogether, we decided to work furtheron with the $1 \%$ profiles and the resulting networks.

\section{Revisiting false positives}

The PPVs obtained (see above and Additional File 2) seem to indicate that there is still a considerable number of FP even under the most stringent conditions (1\% highest scoring conserved TFBSs). To explore this a bit further, we determined again the TP, FP, TN and FN rates of our $1 \%$ top-ranked predictions for five TFs (GATA3, MYC, JUN, MAX, FOS), but using now two independent ENCODE ChIPseq data sets for each of these TFs. These ChIPseq data indicate for each of these factors the binding sites that are used in different cell lines, representing different tissues in all these cases. Figure 2 shows for GATA-3 (see Additional file 3 for the remaining TFs), that two independent ChIP data sets yield quite different TP and FP numbers, with relatively little overlap: they have only 176 targets in common and 


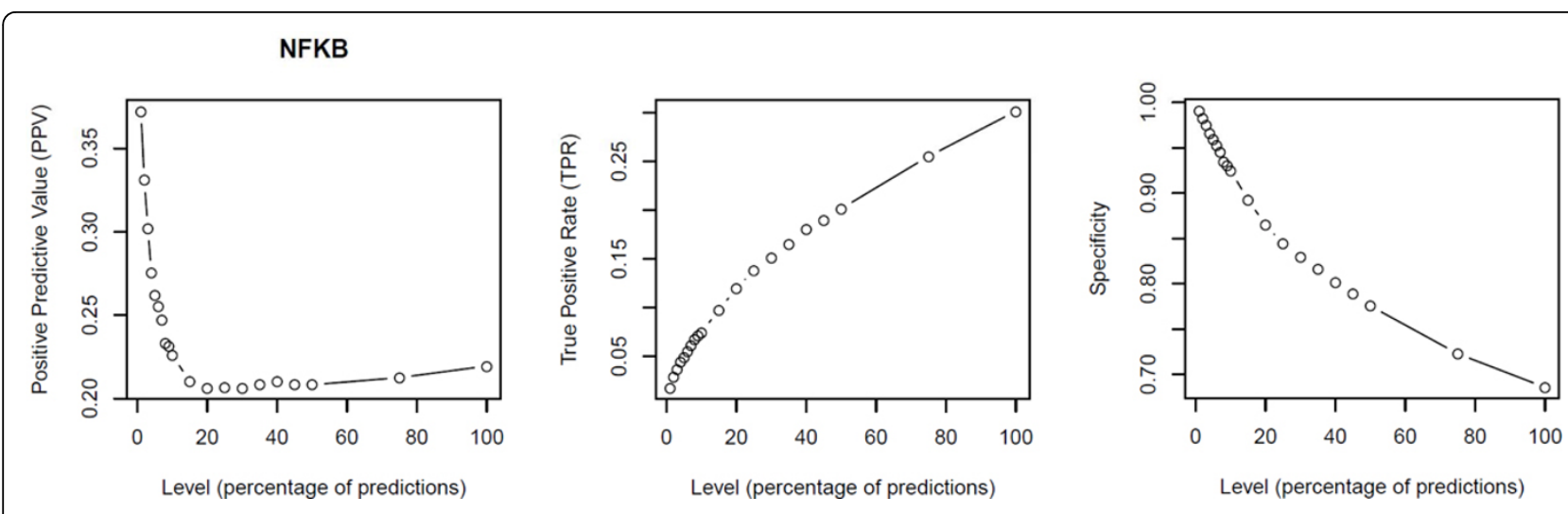

Figure 1 Validation of NF- $\kappa \mathbf{B}$ sites. Validation of predicted NF- $\kappa B$ sites with ChIPseq data sets from ENCODE. Given are the positive predictive value $(P P V=T P /(T P+F P))$, the true positive rate $(T P R=T P /(T P+F N))$, and the specificity $(S P C=T N /(T N+F P))$ for all profiles ranging from the $1 \%$ top-ranking down to $100 \%$ of the predictions made (see Methods for details).

predicted by our approach, and even the overlap between the two experimental data sets comprises only a minority of the proven sites. We obtained the same picture for four further TFs for which we could retrieve new, independent data sets. Altogether, these results clearly show that whatever experimental data set is used for validating the predictions, a considerable number of alleged "false positive" predictions turns into TPs when the experimental basis broadens. In other words, determining the FP rate with only a limited set of experimental data highly overrates this error type.

In the Venn diagram of Figure 2, the overlap between the predictions and any experimental data set may nevertheless appear small when compared with the overlap between the two ChIPseq data sets. It should be noticed, however, that we explicitly accepted a high number of

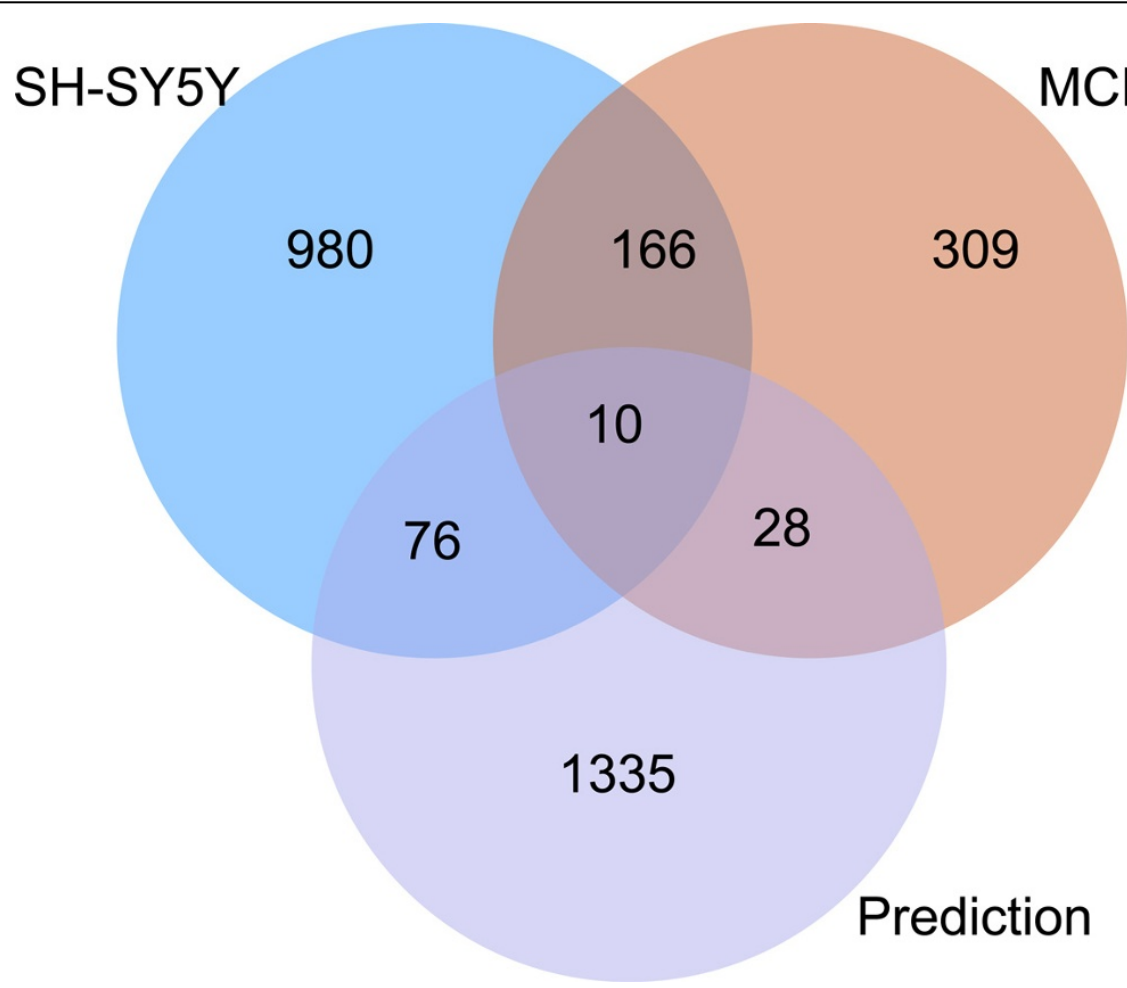

Figure 2 Different ChIPseq data sets show little overlap. Predicted high-affinity (1\% top-ranking), highly conserved binding sites for GATA-3 (purple circle, bottom), and GATA-3 ChIPseq fragments from SH-SY5Y cells (blue circle, top-left) or from MCF7 cells (brown circle, top-right), overlapping with $1 \mathrm{~kb}$ upstream sequences, were checked for the respective intersections. 
False Negatives, as an unavoidable trade-off of the approach chosen here aiming at high-affinity and highly conserved sites only, regarded as potential master regulator or seed sites.

\section{Architecture of the reconstructed network}

For an initial analysis of the reference transcriptional network (RTN) obtained by predicting high-affinity, highly conserved sites and subsequent paralogous expansion, we investigated the distribution of in- and out-degrees. Since especially the out-degrees can adopt very large values, but each degree class is extremely sparsely populated, we computed the inverse cumulative distribution function for the degree frequencies [14]. It was observed that both the RTN as well as the eRTN (expanded reference transcriptional network), when confining to the $1 \%$ highest scoring TFBS, show a clear exponential degree distribution. This is particularly obvious from the corresponding semi-logarithmic plots (Figure 3), where the correlation coefficients for a linear fitting of $1 \%$ profiles are -0.9985 and -0.9982 for the in-degrees and -0.9803 and -0.9846 for the out-degrees (RTN and eRTN, resp.).

Relaxing the prediction constraints, i.e. proceeding from the $1 \%$ to the $100 \%$ profile, reveals the emergence of a shoulder around a degree of 100-200, possible indicating a superposition with a peaked distribution (in-degree) or very heterogeneous distribution until nearly the theoretical maximum (out-degree). This becomes even more visible when relaxing the constraint of conservativity (not shown). More important is that the expanded network (eRTN) in principle shows the same degree distributions, i. e. an exponential degree distribution in the $1 \%$ network (Figure 4). In the out-degree distribution, however, a number of peaks seem to be emerging in the less stringent networks.

\section{Reconstruction of tissue-specific transcription networks}

Previously, we constructed eight tissue-specific transcription networks (TTNs for brain, heart, kidney, liver, ovary, prostate, spleen and testis) by reducing the RTN to those genes that are known to be expressed in the respective tissue [15]. Thus, regulatory edges survive this filtering only if both the regulator and the target gene are found to be expressed in the respective tissue. Here, we reconstructed the transcription networks for the eight tissues based on eRTN where the number of "active" TFs (i.e. those which have an out-degree $>0$ ) has nearly doubled (1.7-fold), and the number of directed edges has nearly tripled (2.6-fold; see above). Compared to the TFs in RTN, which are generally of low tissue-specificity (see Methods), the extra TFs in eRTN are mostly of high tissue-specificity (Figure 5). This indicates that the tissue networks extracted from eRTN are more comprehensive.

\section{Analysis of tissue-specific transcription networks}

In general, the number of TFs in the expanded tissue transcription networks (eTTN) increased on average 1.5 times compared to those in the TTNs, whereas the number of nonTFs is almost constant (Additional file 1). This increase of TF numbers results in an even larger increase in the number of regulations (directed edges), which is on average 2.5 times higher than before the expansion, suggesting that the eTTNs are much more densely connected than the TTNs. It is noted that the increasing ratios of genes and regulations are generally consistent with the reference network and across the different tissues (Additional file 1). This indicates that the extra TFs in the eRTN, which are highly tissue specific (Figure 5), are a characteristic of all tissues studied so far.

The individual eTTN differ considerably in their sizes. By far the largest is the brain network, comprising $75 \%$ of the TF genes, $78 \%$ of the nonTF genes and $61 \%$ of the edges of the eRTN. At the other end of the scale, the spleen network shares with the eRTN only $31 \%$ of the TF genes, $38 \%$ of the nonTF genes, and $11 \%$ of the edges. On average, $41 \%$ of the regulations represented in the eRTN survive the tissue-specific filtering.

As to be expected, the average in-degrees of TFs and nonTFs increase much more than the average out-degrees of TFs in the eTTNs compared with the TTNs. On average, the in-degree rises around 2.3 times, but the outdegree increases only about 1.6 times (Figure 6; see also Additional file 4 for detailed numbers). This is understandable since many TFs are added in the eTTNs, which consequently results in a larger number of regulations that each target gene receives. The moderate increase of the out-degree is due to the fact that most of the newly added TFs had an out-degree well above the average.

Interestingly, the in-degree of TF genes is consistently about $50 \%$ larger than that of nonTF genes. This is true for the (e)RTN as well as for all (e)TTNs. This difference is only slightly diminished by the paralogous expansion (see Additional file 4).

However, such global increase of in- and out-degrees does not change the features of degree distributions of the eTTNs, which all show an exponential distribution of both in- and out-degree (Additional file 5).

\section{Case study on the heart-specific transcription network}

It has been reported that during heart development, T-box transcription factors play a particularly important role [16]. Mutations in human TBX genes may result in cardiovascular malformations. Their gene products, the TBX factors, form a complex spatio-temporal pattern defining the identity of the different heart structures [17].

Human TBX factors are spread over five families, one of them comprising TBX2, TBX3, TBX4 and TBX5 


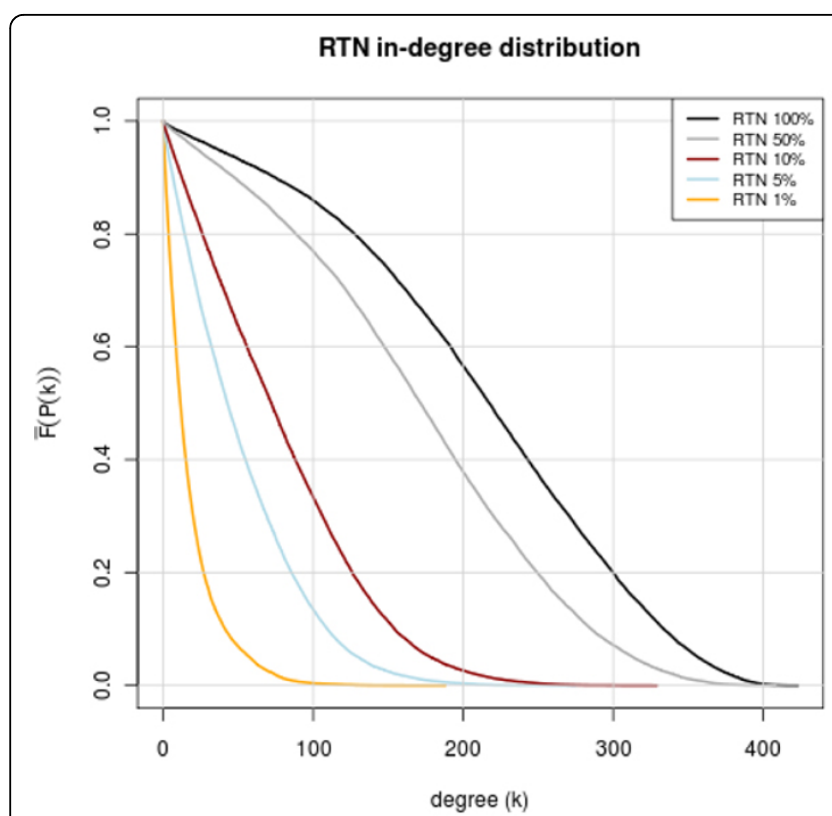

RTN in-degree distribution

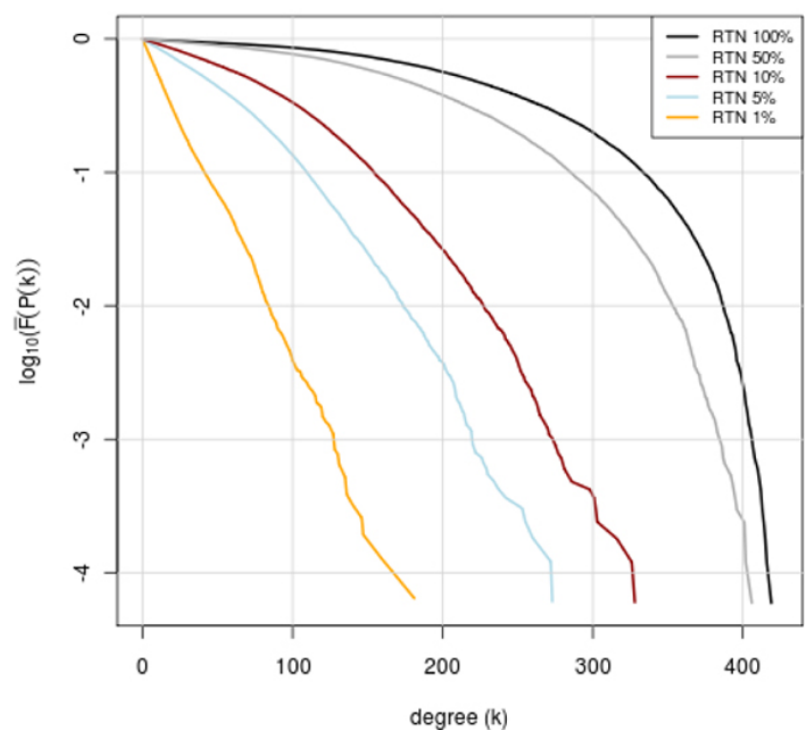

RTN out-degree distribution

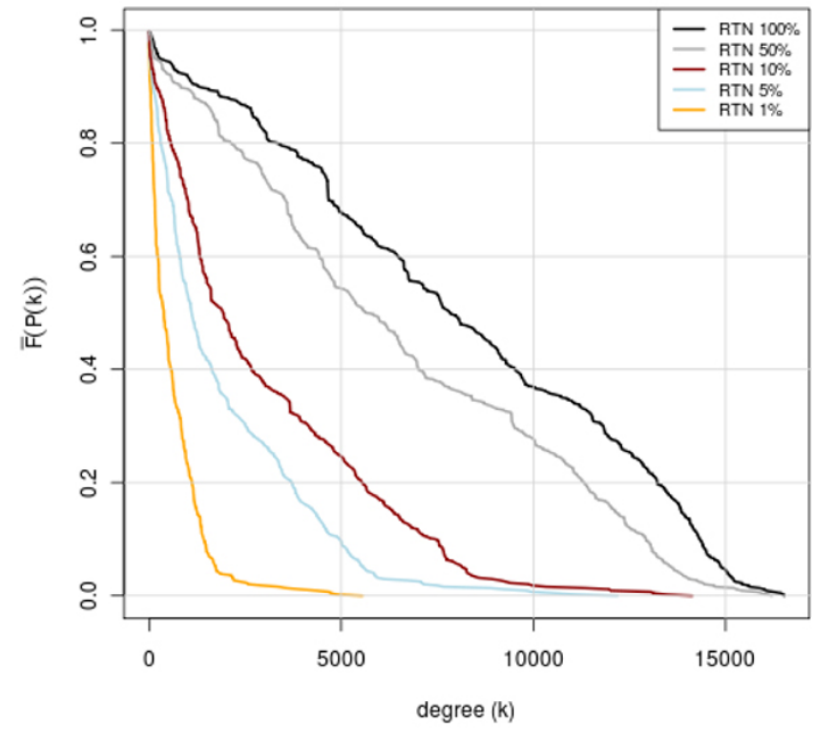

RTN out-degree distribution

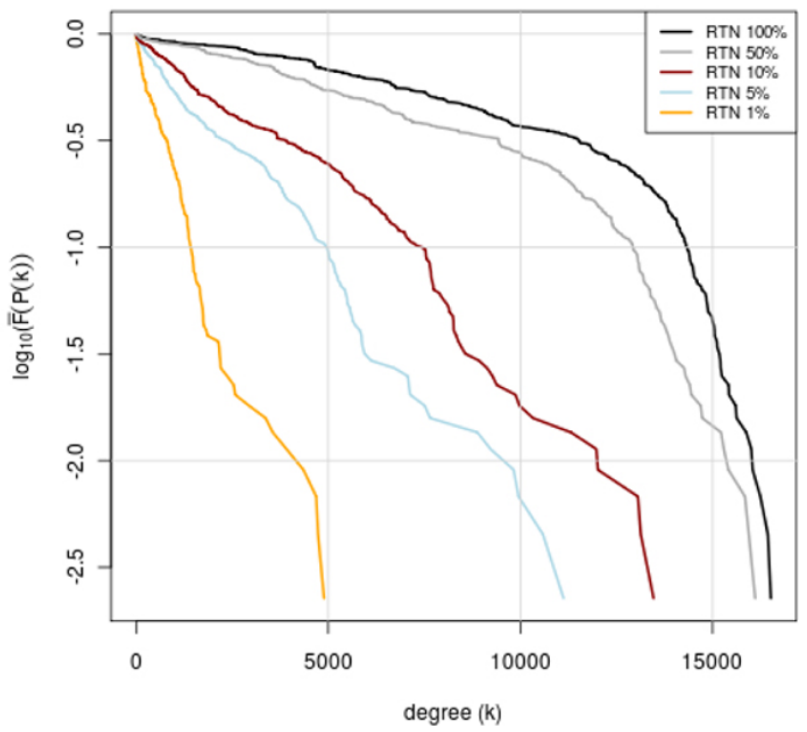

Figure 3 Degree distributions in the reference transcriptional networks (RTNs) Inverse cumulative distribution of in- (left) and outdegree (right), in linear (top) and semi-logarithmic plot (bottom). The distributions are shown for the reference transcriptional networks (RTN) reconstructed with the indicated percentiles of top-ranking predicted and conserved TFBSs.

(family 6.5.4 in our TF classification). Out of them, only TBX5 is associated with a positional weight matrix in TRANSFAC. However, it has been reported that for instance TBX3 can assist pluripotent reprogramming of embryonal fibroblasts, and is required to specify the atrioventricular system (AV) [18]. It prevents genes that are markers for other parts of the organ (e.g., for the chamber myocardium) to be expressed in $\mathrm{AV}$, one of them is the gene of the atrial natriuretic factor (NPPA) [19]. It is noteworthy that after paralogous expansion, our heart network reveals NPPA as one of the more than 2000 target genes of TBX3, a relation that would have been lost otherwise.

\section{Discussion}

With the efforts described in this paper, we made an attempt to reconstruct a realistic transcriptional network that (1) is void of false positive TF-target relations to the utmost extent possible, (2) includes as many regulator nodes (TFs) as possible, and (3) therefore provides a 

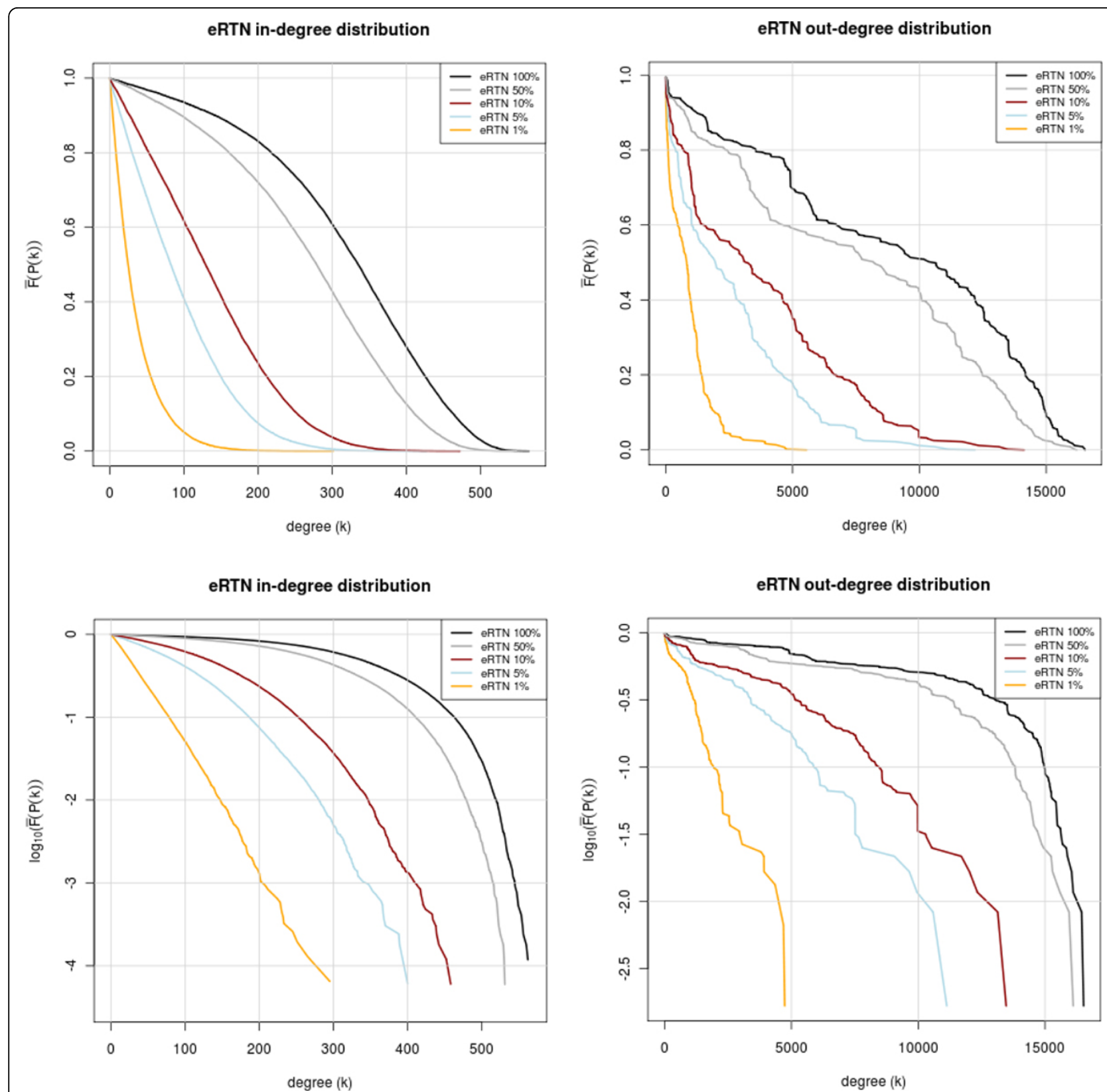

Figure 4 Degree distributions in the expanded reference transcriptional networks (eRTNs). Inverse cumulative distribution of in- (left) and out-degree (right), in linear (top) and semi-logarithmic plot (bottom). The distributions are shown for the expanded reference transcriptional networks (eRTN) reconstructed with the indicated percentiles of top-ranking predicted and conserved TFBSs.

reasonable basis to reconstruct tissue-specific transcriptional networks. In order to minimize the number of false positive predictions, which is a well-known problem in computationally identifying TFBSs, we focused on highly conserved and high-affinity (by virtue of Match score) binding sites only to identify TF-target relations represented by the arcs in our reference network. Since we obtained relatively high PPV for most TFs, we are confident that the network we obtained is reliable. This is supported further by the observation that the FP rates we determined by comparing our predictions with experimental data sets, which always represent one (or very few) specific cellular situation(s), are highly overrated. Comparing experimental data sets for one and the same TF, but obtained from different cell types generally revealed minimal overlaps, confirming that many alleged FPs in fact may turn into true positives in a different cellular context, so that FP numbers are usually overrated. Rather, we suppose that most, if not all, high-affinity and conserved predicted 


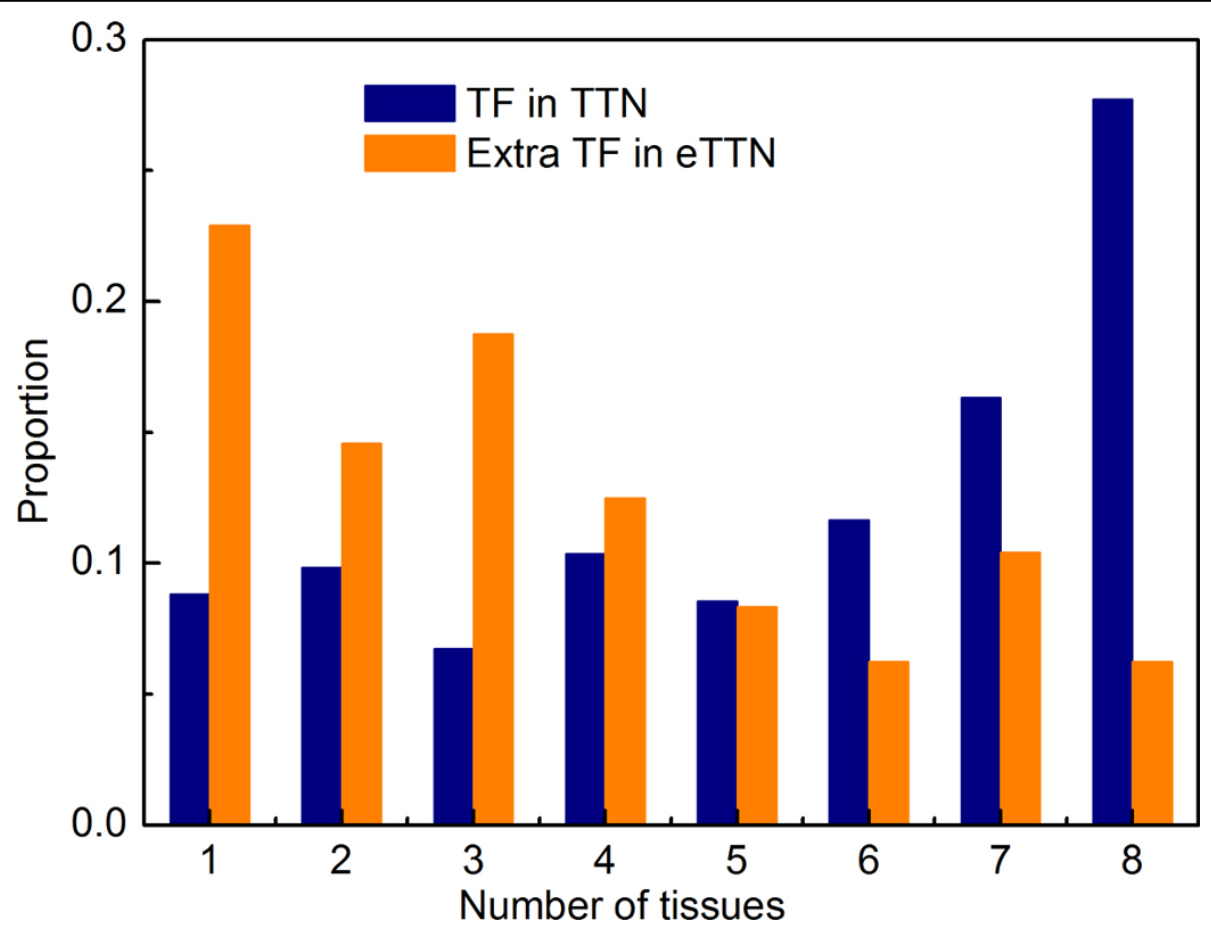

Figure 5 Tissue distribution of the TFs in the tissue-specific transcriptional networks (TTNs) and the corresponding expanded networks (eTTNs). Shown are the portions of TF genes that appear in the indicated numbers of tissue-specific networks. The majority of TFs in the tissue-specific transcriptional networks (TTFs) that were derived from the RTN are expressed in many, if not all tissues studied (blue bars). In contrast, the newly added TF paralogs in the expanded TTNs (eTTNs) show a much more tissue-specific behaviour in being expressed in only one or few tissues (yellow bars).

TFBSs provide a regulatory potential that might be used in a certain cellular situation.

We are aware that our very stringent approach results in large numbers of false negatives, since many experimentally validated TFBS have a very low Match score and gain their functionality by the proper context of other elements. To include this kind of context, or the proper "syntax" of promoters, will be subject of further studies and an according updating of our network. Also the inclusion of enhancers will be a task for future work. We have observed that inclusion of conservativity as criterion does not well apply to enhancer regions, so that new concepts have to be developed for their identification and characterization.

Altogether, we are confident that the networks we have reconstructed reflect a relevant part of reality. This is also supported by the observed kind of degree distribution of the most stringent network, which follows a clear exponential law, as was to be expected at least for the indegree distribution (see [20] and references cited therein) and from our own earlier observations for the out-degree distribution as well [21]. Relaxing the filter criteria leads to degree distributions with more random characteristics.

We have also shown that on the basis of such restrictive filtering, the networks can be reliably expanded by including related TFs and allow them to inherit all target relations, and with that the full out-degree, of already characterized (sub)family members. Since these newly added regulators predominantly provide tissue-specific regulatory information, this expanded network is a good basis to construct reliable transcriptional networks for individual tissues. A first overview revealed for these networks that their degree distributions follow the same rules as the reference network. In addition, first investigations have shown that also the hub composition of all these networks was comparable. Finally, we could show that in the particular case of heart development, paralogous expansion was able to rescue target genes for a specific transcription factor (TBX3), which otherwise would not have been amenable in the corresponding tissue-specific network.

\section{Conclusions}

A paralog-expanded transcriptional network has been constructed based on the knowledge of master regulator or seed sites. It has been shown that the paralogous expansion provides as reliable basis to reconstruct tissue-specific transcription networks. The obtained networks show the expected statistical and topological characteristics. A first case study additionally provided biological evidence for 


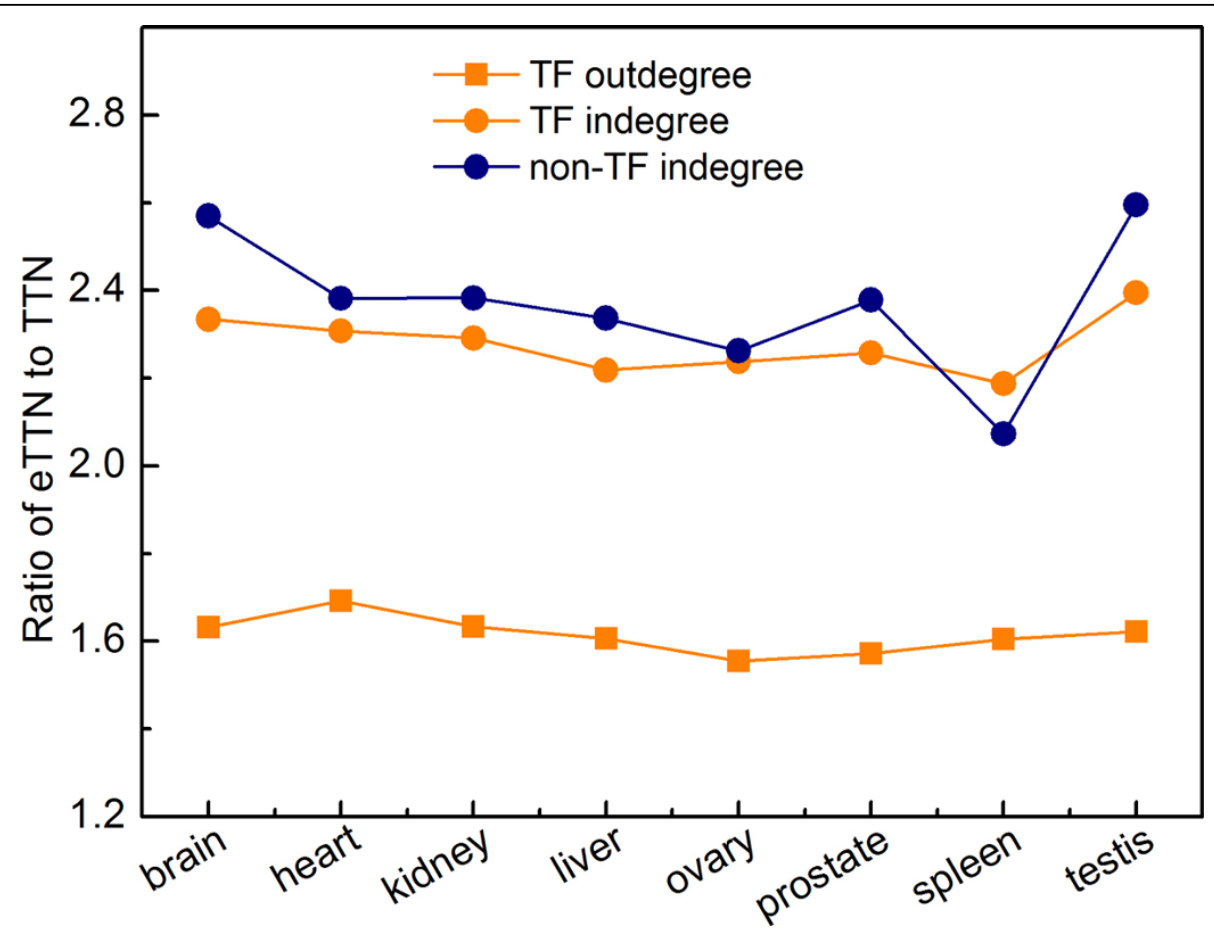

Figure 6 Change of distribution in the tissue-specific networks by paralogous expansion. The ratio of the respective degrees in the expanded tissue-specific networks (eTTNs) to those in the TTNs is shown for the individual tissues. The out-degree of TF genes changed moderately by the expansion in a nearly identical manner across all tissues (about 1.6-fold; yellow squares). In contrast, the in-degree increased considerably (about 2.3-fold), again similarly in all eTTNs (yellow dots). In general, the in-degree of non-TF genes increased slightly more, but with outliers for spleen (below) and testis (above the average; blue dots).

the reliability and usefulness of these networks in including regulatory information which would have been missed without this expansion. From that we conclude that our approach to construct transcriptional network is valid and provides a solid ground for further studies, in particular with regard to the analysis of regulatory processes, e.g. the mechanisms governing cell differentiation.

\section{Additional material}

Additional file 1: Network statistics. Given are the numbers of vertices (split by TF and nonTF genes) and edges in the transcriptional networks without or with expansion, for the reference network as well for the tissue-specific networks.

Additional file 2: Prediction validations. In this file, plots of positive predictive value (PPV), true positive rate (TPR) and Specificity are given for all TFs where sufficient experimental data have been made available by the ENCODE project. Validation has been made in each case for all prediction profiles from the $1 \%$ top-ranking sites down to $100 \%$ of the predictions for conserved binding sites.

Additional file 3: Revisited false positives. Venn diagrams of comparing independent experimental datasets for TFBSs within the $-1 \mathrm{~kb}$ regions with each other and the predictions done in this study.

Additional file 4: Degree statistics. The table indicates the average out-degree and in-degree of TF genes as well as the average in-degree of nonTF genes (NTF) for both the reconstructed transcriptional networks (reference network and tissue-specific networks) as well as the transcriptional network expanded by related TFs. It also shows the ratios of the corrresponding values for the expanded and the non-expanded networks (eTN/TN).

Additional file 5: Degree distributions of tissue-specific transcriptional networks. Inverse cumulative in- and out-degree distributions of the tissue-specific transcriptional networks (TTNs) before and after paralogous expansions.

\section{List of abbreviations}

ChIP: chromatin immunoprecipitation; eRTN: expanded reference transcriptional network; eTTN: expanded tissue-specific transcriptional network; FN: false negative; FP: false positive; PPV: positive predictive value; PWM: positional weight matrix; RTN: reference transcriptional network; TF: transcription factor; TFBS: transcription factor binding site; TN: true negative; TP: true positive; TTN: tissue-specific transcriptional network.

\section{Acknowledgements}

The authors acknowledge the financial support by the European Commission under FP7 (contract no. 202272, LipidomicNet).

This article has been published as part of BMC Systems Biology Volume 6 Supplement 2, 2012: Proceedings of the 23rd International Conference on Genome Informatics (GIW 2012). The full contents of the supplement are available online at http://www.biomedcentral.com/bmcsystbiol/supplements/ $6 / 52$.

\section{Authors' contributions}

$\mathrm{MH}$ carried out the computational analysis of transcription factor binding sites, constructed the reference networks, did the network analyses and drafted parts of the manuscript. $J$ carried out the construction of the tissuespecific transcriptional networks and their analyses and drafted parts of the manuscript. EW conceived of the study, participated in its design and 
coordination and drafted the manuscript. All authors read and approved the final manuscript.

\section{Competing interests}

The authors declare that they have no competing interests.

Published: 12 December 2012

\section{References}

1. Wingender E: The TRANSFAC project as an example of framework technology that supports the analysis of genomic regulation. Brief Bioinform 2008, 9:326-332.

2. Alamanova D, Stegmaier $P$, Kel A: Creating PWMs of transcription factors using $3 \mathrm{D}$ structure-based computation of protein-DNA free binding energies. BMC Bioinformatics 2010, 11:225.

3. Gabdoulline R, Eckweiler D, Kel A, Stegmaier P: 3DTF: a web server for predicting transcription factor PWMs using 3D structure-based energy calculations. Nucleic Acids Res 2012, 40:W180-W185.

4. Heintzman ND, Stuart RK, Hon G, Fu Y, Ching CW, Hawkins RD, Barrera LO, Van Calcar S, Qu C, Ching KA, Wang W, Weng Z, Green RD, Crawford GE, Ren B: Distinct and predictive chromatin signatures of transcriptional promoters and enhancers in the human genome. Nat Genet 2007, 39:311-318.

5. Mullen AC, Orlando DA, Newman JJ, Lovén J, Kumar RM, Bilodeau S, Reddy J, Guenther MG, DeKoter RP, Young RA: Master transcription factors determine cell-type-specific responses to TGF- $\beta$ signaling. Cell 2011, 147:565-576.

6. Zaret KS, Carroll JS: Pioneer transcription factors: establishing competence for gene expression. Genes Dev 2011, 25:2227-2241.

7. Blankenberg D, Taylor J, Nekrutenko A, The Galaxy Team: Making whole genome multiple alignments usable for biologists. Bioinformatics 2011 27:2426-2428.

8. Kel AE, Gössling E, Reuter I, Cheremushkin E, Kel-Margoulis OV, Wingender E: MATCH: A tool for searching transcription factor binding sites in DNA sequences. Nucleic Acids Res 2003, 31:3576-3579.

9. ENCODE Project Consortium: A user's guide to the encyclopedia of DNA elements (ENCODE). PLoS Biol 2011, 9:e1001046.

10. Wheeler DL, Church DM, Federhen S, Lash AE, Madden TL, Pontius JU, Schuler GD, Schriml LM, Sequeira E, Tatusova TA, Wagner L: Database resources of the National Center for Biotechnology. Nucleic Acids Res 2003, 31:28-33.

11. Sauer $T$, Shelest $E$, Wingender E: Evaluating phylogenetic footprinting for human-rodent comparisons. Bioinformatics 2006, 22:430-437.

12. Xie X, Rigor P, Baldi P: MotifMap: a human genome-wide map of candidate regulatory motif sites. Bioinformatics 2009, 25:167-174.

13. Heinemeyer T, Chen X, Karas H, Kel AE, Kel OV, Liebich I, Meinhardt T, Reuter I, Schacherer F, Wingender E: Expanding the TRANSFAC database towards an expert system of regulatory molecular mechanisms. Nucleic Acids Res 1999, 27:318-322.

14. Tanaka R, Yi TM, Doyle J: Some protein interaction data do not exhibit power law statistics. FEBS Lett 2005, 579:5140-5144.

15. Li J, Hua X, Haubrock M, Wang J, Wingender E: The architecture of the gene regulatory networks of different tissues. Bioinformatics 2012, 28 : i509-i514.

16. Plageman TF Jr, Yutzey KE: T-box genes and heart development: putting the "T" in heart. Dev Dyn 2005, 232:11-20.

17. Greulich F, Rudat C, Kispert A: Mechanisms of T-box gene function in the developing heart. Cardiovasc Res 2011, 91:212-222.

18. Han J, Yuan P, Yang H, Zhang J, Soh BS, Li P, Lim SL, Cao S, Tay J, Orlov YL, Lufkin T, Ng HH, Tam WL, Lim B: Tbx3 improves the germ-line competency of induced pluripotent stem cells. Nature 2010, 463:1096-1100.

19. Bakker ML, Boukens BJ, Mommersteeg MT, Brons JF, Wakker V, Moorman AF, Christoffels VM: Transcription factor Tbx3 is required for the specification of the atrioventricular conduction system. Circ Res 2008, 102:1340-1349.

20. Martinez NJ, Walhout AJ: The interplay between transcription factors and microRNAs in genome-scale regulatory networks. Bioessays 2009, 31:435-445.
21. Goemann B, Wingender E, Potapov AP: Topological peculiarities of mammalian networks with different functionalities: transcription, signal transduction and metabolic networks. Network Biol 2011, 1:134-148.

doi:10.1186/1752-0509-6-S2-S15

Cite this article as: Haubrock et al:: Using potential master regulator sites and paralogous expansion to construct tissue-specific transcriptional networks. BMC Systems Biology 2012 6(Suppl 2):S15.

\section{Submit your next manuscript to BioMed Central and take full advantage of:}

- Convenient online submission

- Thorough peer review

- No space constraints or color figure charges

- Immediate publication on acceptance

- Inclusion in PubMed, CAS, Scopus and Google Scholar

- Research which is freely available for redistribution

Submit your manuscript at www.biomedcentral.com/submit
Biomed Central 